\title{
Act requires officials to review how US dietary guidelines are formulated
}

In this News story (BMJ 2015;351:h6930, doi:10.1136/bmj. h6930), we said that the new US dietary guidelines, due to be published this month, would recommend that people should "reduce the total energy in their diet from saturated fat to $10 \%$, down from $30 \%$ in previous guidelines."

It was incorrect to say that the previous guidelines recommended that people should not consume more than $30 \%$ of their energy from saturated fat. The previous 2010 guidelines had recommended that total fat intake — not saturated fat intake- - be kept between 20 to $35 \%$ of calories. In a shift, the new Dietary Guidelines Advisory Committee scientific report recommended that the new guidelines "should put the emphasis on optimizing types of dietary fat and not reducing total fat."

We apologise for this error.

Cite this as: BMJ 2016;352:162

๑ BMJ Publishing Group Ltd 2016 